Este trabalho se destacou entre as apresentações orais, na categoria tema livre, do I Congresso Internacional de Fisioterapia em Pelviperineologia, contendo $\circ \mathrm{X}$ Encontro Nordestino de Fisioterapia na Saúde da Mulher e o III Encontro Nordestino de Fisioterapia na Saúde do Homem.

\title{
AVALIAÇÃO DA FUNÇÃO SEXUAL EM PRIMÍPARAS APÓS PARTO VAGINAL E NULIGESTAS
}

\section{EVALUATION OF THE SEXUAL FUNCTIONS IN PRIMIPARAS AFTER VAGINAL DELIVERY AND NULLIGRAVIDAS}

Lorena Carneiro de Macêdo', Hellen Batista de Carvalho², Suyane Waneide Macêdo de Medeiros ${ }^{3}$, Andressa Maria Bezerra dos Santos ${ }^{3}$, Leila Katz ${ }^{4}$, Melania Maria Ramos de Amorim ${ }^{5}$

\section{Rédica. Doutora em Tocoginecologia. $\begin{aligned} & \text { Docente do Instituto de Medici } \\ & \text { (UFCG). Pesquisadora pelo Ins }\end{aligned}$
RESUMO I Introdução: a gestação e o parto podem
prejudicar a função dos músculos do assoalho pélvico (MAP) e influenciar na função sexual após o parto. Objetivo: avaliar a função sexual em primíparas pós-parto vaginal e em nuligestas. Materiais e Métodos: estudo transversal descritivo, aprovado pelo Comitê de Ética em Pesquisa da UEPB (CAAE44775015.1.0000.5175) realizado com 36 mulheres, faixa etária de 18 a 35 anos, sendo primíparas pós-parto vaginal com episiotomia $(n=12)$, sem episiotomia $(n=12)$ e mulheres nuligestas $(n=12)$. Foram verificadas características biológicas, sociodemográficas e avaliada a função sexual através do Female Sexual Function Index (FSFI). As primíparas responderam ao questionário três meses após o parto e o mesmo questionário foi respondido pelas nuligestas. Resultados: as mulheres com episiotomia, sem episiotomia e nuligestas apresentaram média de $21,41 \pm 4,56 ; 20,16 \pm 4,60$ e $26,3 \pm 4,16$ anos de idade, média de $14,66 \pm 2,42 ; 14,81 \pm 2,44$ e $18,83 \pm 1,80$ anos estudados, e frequência de dispareunia de $66,66 \%(n=8)$; $50 \%(n=6)$ e $0 \%(n=0)$, respectivamente. Em relação a função sexual, ao comparar os três grupos da pesquisa, foi encontrado um menor escore de desejo nas primíparas com episiotomia e o grupo sem episiotomia apresentou menos excitação, menos lubrificação, menos orgasmos e uma pior satisfação sexual, quando comparado aos outros grupos. Quanto a variável dor, as primíparas com episiotomia apresentaram os piores escores. Conclusão: no grupo de primíparas com episiotomia, foi encontrada maior frequência de dispareunia, índices mais baixos de desejo e as primíparas sem episiotomia apresentaram menos excitação, menos lubrificação, menos orgasmos e uma pior satisfação sexual.}

\begin{abstract}
Introduction: Pregnancy and delivery can impair the function of pelvic floor muscles (PFM) and influence sexual function after delivery. Objective: To evaluate sexual function in primiparas after vaginal delivery and nulligravidas. Materials and Methods: A descriptive cross-sectional study, approved by the Ethics Committee on Research (CAAE44775015.1.0000.5175), and carried out with 36 women aged 18-35 years, being post-vaginal delivery primiparas with episiotomy $(n=12)$, without episiotomy $(n=12)$ and nulligravidas $(n=12)$. Biological and sociodemographic characteristics were verified and the sexual function was evaluated through the Female Sexual Function Index (FSFI). The primiparas answered the questionnaire three months after the delivery and the same questionnaire was answered by the nulligravidas. Results: Women with episiotomy, without episiotomy and nulligravidas presented an average of $21.41 \pm 4.56$; $20.16 \pm 4.60$ and $26.3 \pm 4.16$ years old, average of $14.66 \pm 2.42 ; 14.81 \pm 2.44$ and $18.83 \pm 1.80$ studied years, and dyspareunia frequency of $66.66 \%(n=8)$; $50 \%(n=6)$ and $0 \%(n=0)$, respectively. In relation to sexual function, when comparing the three groups of the research, a lower desire score was found in primiparas with episiotomy, and the non-episiotomy group presented less excitation, less lubrication, fewer orgasms and worse sexual satisfaction when compared to the other groups. Regarding the variable pain, the primiparas with episiotomy presented the worst scores. Conclusion: In the group of primiparas with episiotomy, a higher frequency of dyspareunia was found, lower rates of desire and primiparas without episiotomy had less excitement, less lubrication, less orgasms and worse sexual satisfaction.
\end{abstract}

Keywords: Postpartum period. Pelvic floor. Episiotomy.

Palavras-chave: Período pós-parto. Assoalho pélvico. Episiotomia. 


\section{INTRODUÇÃO}

A gestação, o parto e o puerpério são períodos de mudanças físicas e psicológicas para a mulher. $\mathrm{Na}$ gestação, os músculos do assoalho pélvico (MAP) sofrem sobrecarga constante devido ao crescimento uterino e durante $o$ terceiro trimestre gestacional podem sofrer sobrecarga ocasionada pelo encaixamento e pela progressão da cabeça fetal. Além disso, as estratégias neuromusculares para controle postural durante a gestação, utilizadas pela mulher ao realizar suas atividades, podem contribuir para alterações miofasciais do assoalho pélvico ${ }^{1,2}$.

No momento do parto, o assoalho pélvico fica exposto à compressão do feto e às pressões para baixo devido aos esforços expulsivos realizados pela parturiente. Estas forças distendem o assoalho pélvico, resultando em alterações anatômicas e funcionais nos músculos, nervos e tecidos conectivos. Danos à inervação do elevador do ânus e dos músculos esfincterianos têm sido associados à diminuição da força muscular depois do parto ${ }^{3,4}$.

No pós-parto, os MAP podem apresentar-se mais hipotônicos e distendidos pela ação hormonal, pela sobrecarga do bebê e por possíveis traumas durante - trabalho de parto (lacerações espontâneas ou episiotomia). Nesse período, a vagina pode se apresentar sensível e ressecada, o que pode ocasionar disfunção sexual pós-parto em algumas mulheres ${ }^{5}$.

Possuindo uma prevalência que varia de 20 a $73 \%$ nas mulheres, as disfunções sexuais femininas (DSF) resultam da combinação de fatores biológicos, psicológicos, sociais e culturais, resultando em um bloqueio total ou parcial da resposta sexual do indivíduo, relacionada ao desejo, a excitação e ao orgasmo 6 . Durante o período da gestação e pós-parto, existe um aumento na ocorrência das disfunções sexuais, com prevalências de 33,5\% antes da gestação, $76 \%$ durante a gestação e $43,5 \%$ no pós-parto 7 .

Um estudo realizado para investigar a vivência da sexualidade no puerpério verificou insatisfação dessas mulheres com $\circ$ ato sexual, devido a queixas como dispareunia, gerada por doenças ginecológicas, infecções, distúrbios hormonais e falta de lubrificação vaginal ${ }^{8}$.

Dessa forma, o objetivo do presente estudo foi avaliar a função sexual em primíparas após o parto vaginal e em nuligestas.

\section{MÉTODOS}

O estudo de corte transversal foi realizado no Instituto de Pesquisa Professor Joqquim Amorim Neto (IPESQ), localizado em Campina Grande/PB, com amostra de 36 mulheres com faixa etária de 18 a 35 anos, divididas em primíparas pós-parto vaginal com episiotomia $(n=12)$, primíparas pósparto vaginal sem episiotomia $(n=12)$ e nuligestas $(n=12)$.

Para participar da pesquisa, as nuligestas deveriam ter 18 anos ou mais, vida sexual iniciada e ausência de gestação prévia com duração maior que três meses e foram considerados como critérios de exclusão: história prévia de cirurgia abdominal ou urogenital e presença de prolapso genital.

Já as primíparas possuíam como critérios de inclusão ser primeira gestação, faixa etária de 18 a 39 anos, gestação a termo ( 37 a 42 semanas) e recémnascido vivo. $E$ foram considerados critérios de exclusão: gestação de alto risco, parto instrumental, gestação gemelar, história prévia de cirurgia abdominal ou urogenital e presença de prolapso genital.

As primíparas foram captadas no Instituto de Saúde Elpídio de Almeida (ISEA), Campina Grande/PB, durante $o$ pós-parto imediato. Nesse contato inicial foram explicados os objetivos e procedimentos da pesquisa, e as mulheres que concordaram em participar, assinaram o Termo de Consentimento Livre e Esclarecido (TCLE) para que em seguida, fossem coletadas informações de identificação, endereço e contato das participantes. Três meses depois do parto, as primíparas elegíveis foram contatadas 
e compareceram ao IPESQ para realização de entrevista com o preenchimento do formulário de pesquisa, que contém informações biológicas e sociodemográficas, hábitos de vida e características da vida sexual, e da função sexual.

As nuligestas foram recrutadas por meio de uma busca ativa nas Instituições de Ensino Superior da cidade de Campina Grande, onde foram informadas sobre os objetivos da pesquisa, concordando em participar, assinaram o Termo de Consentimento Livre e Esclarecido (TCLE) e compareceram ao IPESQ para coleta de dados com realização da entrevista, respondendo ao mesmo formulário de pesquisa aplicado às primíparas.

A avaliação da função sexual foi realizada através do questionário Female Sexual Function Index (FSFI), com versão validada para o português ${ }^{9}$, que apresenta 19 questões que avaliam a função sexual nas últimas quatro semanas, no qual cada resposta recebe uma pontuação de zero a cinco. Um escore total é apresentado no final da aplicação, resultado da soma dos escores de cada domínio multiplicada por um fator numérico pré-determinado que homogeneiza a influência de cada domínio no escore total. Ao avaliar o domínio "dor", quanto menor o escore, pior o grau da dor na relação sexual, sendo pior o desempenho. Enquanto na avaliação dos demais domínios (desejo, excitação, lubrificação vaginal, orgasmo e satisfação sexual) um menor escore corresponde a uma melhor classificação.

O estudo foi aprovado pelo Comitê de Ética em Pesquisa da UEPB (CAAE 44775015.1.0000.5175), sendo obedecidas todas as normas para realização de pesquisas com seres humanos. Todas as mulheres avaliadas que apresentavam alguma disfunção sexual, ao final da avaliação, foram encaminhadas a um serviço público especializado para tratamento da disfunção.

Os dados coletados foram organizados e tabulados, pelos pesquisadores, em planilha eletrônica

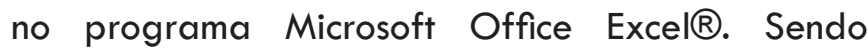
construídas tabelas de distribuição de frequência para exposição dos resultados. Para a análise inferencial foi utilizado o pacote estatístico SPSS versão 20 sendo utilizado o Teste de Kruskal-Wallis, seguido do método Dunn para verificar a diferença entre os grupos.

\section{RESULTADOS}

De acordo com os dados coletados, foi verificado que as nuligestas tinham maior idade, maior número de anos estudados e maior tempo de vida sexualmente ativa ( $9 \pm 4,69$ anos) quando comparado com os grupos de primíparas. Os resultados referentes às características biológicas e sociodemográficas estão representados na tabela 1.

\begin{tabular}{|c|c|c|c|}
\hline Variável & $\begin{array}{c}\text { Com episiotomia } \\
N=12\end{array}$ & $\begin{array}{c}\text { Sem episiotomia } \\
N=12\end{array}$ & $\begin{array}{c}\text { Nuligesta } \\
\mathrm{N}=12\end{array}$ \\
\hline \multicolumn{4}{|l|}{ Idade (anos) } \\
\hline $\mathrm{X} \pm \mathrm{DP}$ & $21,41 \pm 4,56$ & $20,16 \pm 4,60$ & $26,3 \pm 4,16$ \\
\hline Limites (menor - maior) & $16-34$ & $14-30$ & $20-35$ \\
\hline \multicolumn{4}{|l|}{ IMC $\left(\mathbf{k g} / \mathbf{m}^{2}\right)$} \\
\hline $\mathrm{X} \pm \mathrm{DP}$ & $24,94 \pm 3,17$ & $23,77 \pm 5,42$ & $22,94 \pm 2,97$ \\
\hline Limites (menor - maior) & $18,26-28,33$ & $16,91-33,27$ & $17,93-27,66$ \\
\hline \multicolumn{4}{|c|}{ Escolaridade (anos estudados) } \\
\hline$X \pm D P$ & $14,66 \pm 2,42$ & $14,81 \pm 2,44$ & $18,83 \pm 1,80$ \\
\hline Limites (menor - maior) & $10-20$ & $10-19$ & $14-20$ \\
\hline \multicolumn{4}{|l|}{ Tabagismo - n (\%) } \\
\hline Sim & 0 & $1(8,4 \%)$ & 0 \\
\hline Não & $12(100 \%)$ & $11(91,6 \%)$ & $12(100 \%)$ \\
\hline \multicolumn{4}{|l|}{ Etilismo-n (\%) } \\
\hline Sim & 0 & $1(8,4 \%)$ & $1(8,4 \%)$ \\
\hline Não & $12(100 \%)$ & $11(91,6 \%)$ & $11(91,6 \%)$ \\
\hline \multicolumn{4}{|c|}{ Prática de exercício físico ( $n, \%)$} \\
\hline Sim & $1(8,4 \%)$ & 0 & $2(16,67 \%)$ \\
\hline Não & $11(91,6 \%)$ & $12(100 \%)$ & $10(83,33 \%)$ \\
\hline
\end{tabular}


Foi verificado que o grupo de primíparas com episiotomia tem a maior frequência de mulheres sexualmente inativas $(16,6 \%)$ e essas mulheres têm maior frequência de dor sexual pós-parto $(66,6 \%)$.

As características da função sexual de acordo com os escores de avaliação do FSFI (Female Sexual Function Index) estão apresentadas na tabela 2. Comparando os resultados entre os três grupos, foi verificado que as primíparas apresentam pior função sexual em relação às nuligestas, e que as primíparas sem episiotomia apresentam função sexual diminuída na maioria dos domínios (excitação, lubrificação, orgasmo e satisfação) enquanto que as primíparas com episiotomia apresentam pior função em relação ao desejo e maiores escores em relação a dor, de forma que a dor está mais frequente e tem maior gravidade nesse grupo. Embora tenha sido verificada diferença nos valores absolutos das médias avaliadas entre os grupos, não foi encontrada diferença estatisticamente significante $(p>0,05)$.

Tabela 2. Características da função sexual através dos escores de avaliação do FSFI (Female Sexual Function Index). Campina Grande-PB, 2016.

\begin{tabular}{|c|c|c|c|}
\hline Variável & $\begin{array}{c}\text { Com episiotomia } \\
N=12\end{array}$ & $\begin{array}{l}\text { Sem episiołomia } \\
N=12\end{array}$ & $\begin{array}{c}\text { Nulíparas } \\
\mathrm{N}=12\end{array}$ \\
\hline \multicolumn{4}{|l|}{ Desejo } \\
\hline$X \pm D P$ & $4,1 \pm 0,76$ & $3,9 \pm 0,90$ & $3,7 \pm 0,43$ \\
\hline Limites (menor - maior) & $3-5,4$ & $2,4-5,4$ & $3-4,2$ \\
\hline \multicolumn{4}{|l|}{ Excitação } \\
\hline $\mathrm{X} \pm \mathrm{DP}$ & $2,95 \pm 1,34$ & $3,5 \pm 0,88$ & $2,55 \pm 0,75$ \\
\hline Limites (menor - maior) & $0-4,8$ & $2,4-5,4$ & $1,5-3,9$ \\
\hline \multicolumn{4}{|l|}{ Lubrificação } \\
\hline $\mathrm{X} \pm \mathrm{DP}$ & $3,4 \pm 1,40$ & $3,87 \pm 0,86$ & $3,85 \pm 0,38$ \\
\hline Limites (menor - maior) & $0-4,8$ & $2,4-5,4$ & $3,3-4,5$ \\
\hline \multicolumn{4}{|l|}{ Orgasmo } \\
\hline $\mathrm{X} \pm \mathrm{DP}$ & $2,93 \pm 1,22$ & $3,53 \pm 0,47$ & $3,43 \pm 0,49$ \\
\hline Limites (menor - maior) & $0-4,4$ & $2,8-4,4$ & $2,8-4,4$ \\
\hline \multicolumn{4}{|l|}{ Satisfação } \\
\hline$X \pm D P$ & $2,03 \pm 1,13$ & $2,16 \pm 0,79$ & $2,06 \pm 0,56$ \\
\hline Limites (menor - maior) & $0-4$ & $1,2-4,4$ & $1,2-2,8$ \\
\hline \multicolumn{4}{|l|}{ Dor } \\
\hline$X \pm D P$ & $3,6 \pm 1,48$ & $4,83 \pm 0,95$ & $5,56 \pm 0,55$ \\
\hline Limites (menor - maior) & $1,2-6$ & $3,6-6$ & $4,4-6$ \\
\hline \multicolumn{4}{|l|}{ Escore total } \\
\hline $\mathrm{X} \pm \mathrm{DP}$ & $19,01 \pm 5,03$ & $21,80 \pm 3,1$ & $21,16 \pm 1,8$ \\
\hline Limites (menor - maior) & $7,6-23,6$ & $15,6-27,8$ & $18,1-23,7$ \\
\hline
\end{tabular}

\section{DISCUSSÃO}

Os resultados encontrados nesse estudo mostram que as mulheres no pós-parto apresentaram piores índices de função sexual, na maioria dos domínios, quando comparadas com as nuligestas. Não foi verificada diferença estatisticamente significante entre os grupos avaliados $(p>0,05)$ mas houve diferença nos valores absolutos das médias encontradas entre os grupos estudados.

Foi verificado que a maioria das primíparas com episiotomia apresentou dor durante a relação sexual, tendo também piores escores no domínio desejo. A resposta sexual envolve uma sequência coordenada de fases (desejo, excitação, lubrificação, orgasmo e resolução) e todos os componentes da resposta são mutuamente dependentes, sugerindo que a presença da dor possa estar relacionada com a inibição de outros componentes da resposta sexual ${ }^{9}$, o que pode justificar a influência da dispareunia no baixo desejo sexual dessas primíparas com episiotomia.

A dor durante a atividade sexual compromete qualidade de vida, a função sexual da mulher, afetando $\circ$ relacionamento de casais estáveis, pois o parceiro pode evitar o contato com receio de causar esse desconforto, podendo diminuir sua iniciativa para a atividade sexual ou até mesmo diminuir seu interesse ${ }^{10}$. 
Corroborando com nossos achados, um estudo apresentou relevante prevalência de disfunção sexual feminina na gestação e no puerpério, estando presente em cerca de $70 \%$ das gestantes brasileiras ${ }^{11}$ e em $86 \%$ das mulheres no puerpério, sendo as disfunções mais frequentes a dispareunia (22 a $41 \%$ ) e diminuição do desejo sexual (83 a $86 \%)^{12,13}$. No terceiro mês após o parto, 45 a $55 \%$ das mulheres referem dispareunia e, no sexto mês, 18 a $30 \%$ ainda persistem com problemas sexuais $^{14,15}$.

Um estudo desenvolvido em um hospital universitário na Suécia comparou a saúde sexual das mulheres submetidas ou não à episiotomia. Os resultados não mostraram diferença significativa no nível de satisfação sexual entre os grupos, apesar de indicar que as mulheres que tiveram episiotomia apresentaram maior desconforto vaginal, encontrando associação entre a realização de episiotomia e a ocorrência de dispareunia ${ }^{16}$.

Esses achados vêm complementar o conhecimento existente sobre a função sexual no pós-parto. A literatura mostra que mulheres que tiveram parto vaginal com episiotomia apresentaram no pósparto, menores níveis de libido, mais dificuldade para atingir o orgasmo, menor satisfação sexual e maior grau de dor durante a relação sexual comparando com as mulheres com períneo íntegro ou lacerações espontâneas ${ }^{17}$.

Mesmo encontrando menor função sexual em primíparas após o parto vaginal, a operação cesariana não deve ser considerada um fator de proteção para o assoalho pélvico, já que não foi encontrada diferença na função sexual (avaliação de 12 a 18 meses pós-parto) entre mulheres submetidas ao parto normal ou cesariana eletiva ${ }^{18}$.

Ainda nesse contexto, buscando determinar 0 efeito em longo prazo (6-11 anos) do tipo de parto sobre a prevalência e gravidade da dor pélvica (dismenorréia, dispareunia e dor pélvica não relacionados com a menstruação ou a relação sexual) foi verificado que o parto vaginal não está associado a uma maior taxa de dor pélvica, quando comparado com a cesariana, com excessão do parto forcéps e parto vaginal de um bebê com mais de quatro quilos, que têm associação com dispareunia 6-11 anos após o parto normal ${ }^{19}$.

\section{CONCLUSÃO}

Primíparas depois do parto vaginal apresentaram pior função sexual em relação às nuligestas, tendo as primíparas com episiotomia pior função em relação ao desejo e maiores escores em relação a dor sexual, enquanto as primíparas sem episiotomia apresentaram função sexual diminuída na excitação, lubrificação, orgasmo e satisfação.

Vale salientar que outros estudos devem ser realizados com uma amostra maior, avaliando mulheres depois da cesárea e correlacionando os achados da função sexual com as características do parto e do nascimento.

\section{CONTRIBUIÇÕES DOS AUTORES}

Macêdo LC e Carvalho HB foram responsáveis por contribuições substanciais para a concepção e design, e aquisição de dados; Medeiros SWM e Santos AMB foram responsáveis por contribuições para a aquisição de dados. Amorim MMR e Katz L foram responsáveis por contribuições na elaboração do artigo e revisão crítica, aprovação final da versão a ser submetida e das versões revisadas.

\section{CONFLITO DE INTERESSES}

Nenhum conflito financeiro, legal ou político envolvendo terceiros (governo, empresas e fundações privadas, etc.) foi declarado para nenhum aspecto do trabalho submetido (incluindo mas não limitandose a subvenções e financiamentos, conselho consultivo, desenho de estudo, preparação de manuscrito, análise estatística, etc).

\section{REFERÊNCIAS}

1. Pereira SB. Impacto do parto na atividade eletromiográfica do assoalho pélvico e nos sintomas do trato urinário inferior: estudo prospectivo comparativo [Doutorado]. Universidade Estadual de Campinas: 2008

2. Lee DG, Lee LJ, McLaughlin L. Stability, Continence and Breathing: The Role of Fascia Following Pregnancy and Delivery. Journal of Bodywork and Movement Therapies. 2008;1 2:333-48. doi: 10.1016/i.jbmt.2008.05.003

3. Moreno AL. Fisioterapia Em Uroginecologia. $2 a$ ed. Barueri, SP: Manole; 2009

4. Handa VL, Harris TA, Ostergard DR. Protecting the pelvic floor: obstetric management to prevent incontinence and 
pelvic organ prolapse. Obstet Gynecol. 1996;88(3):470-8. doi: 10.1016/0029-7844(96)00151-2

5. Lemos A. Fisioterapia obstétrica baseada em evidências. Rio de Janeiro: Medbook, 2014

6. Holanda JBL, Abuchaim ESV, Coca KP, Abrão ACFV. Disfunção sexual e fatores associados relatados no período pós-parto. Acta Paul Enferm. 2014; 27(6):573-8. doi: 10.1590/1982- 0194201400093

7. Mathias AERA, Pitangui ACR, Arantes VA, Freitas HGV, Vilela FMF, Dias TG. Disfunção sexual: Avaliação de mulheres durante o terceiro trimestre gestacional. ABCS Health Sci. 2015; 40(2):75-9. doi: 10.7322/abcshs.v40i2.734

8. Salim NR, Gualda DM. Sexuality in the puerperium: the experience of a group of womem. Rev. Esc. Enferm. 2010; 44: 88-95. doi: 10.1590/S0080-62342010000400005

9. Pacagnella RC, Martinez EZ, Vieira EM. Validação de constructo de uma versão em português do Female Sexual Function Index. Cad. Saúde Pública. 2009; 25: 2333-44. doi: $10.1590 /$ S0102-311X2009001100004

10. Abdo CHN, Fleury HJ. Dor genital feminina. Diagnóstico e Tratamento. 2013; 18: 124-7

11. Ribeiro MC, Nakamura MU, Abdo CHN, Torloni MR, Scanavino MT, Mattas R. Gravidez e Diabetes Gestacional: uma combinação prejudicial à função sexual feminina? Rev Bras Ginecol Obstet. 201 1;33(5):219-24. doi: 10.1590/ S0100-72032011000500003

12. Hicks TL, Goodall SF, Quattrone EM, Lydon-Rochelle MT. Postpartum sexual functioning and method of delivery: summary of the evidence. J Midwifery Womens Health. 2004;49(5):430-6. doi: 10.1016/i.jmwh.2004.04.007

13. Acele EÖ, Karaçam Z. Sexual problems in women during the first postpartum year and related conditions. J Clin Nurs. 201 2;21 (7-8):929-37. doi: $10.1111 /$ i.13652702.201 1.03882.x

14. Barrett G, Pendry E, Peacock J, Victor C, Thakar R, Manyonda I. Women's sexual health afterchild birth. Br J Obstet Gynaecol. 2000;107(2):186-95

15. Rogers RG, Borders N, Leeman LM, Albers LL. Does spontaneous genital tract trauma impact postpartum sexual function? Midwifery Womens Health. 2009; 54(2):98-103. doi: 10.1016/i.jmwh.2008.09.001

16. Ejegard H, Ryding EL, Sjogren B. Sexuality after delivery with episiotomy: a long-term follow-up. Gynecol Obstet Invest. 2008;66(1):1-7. doi: $10.1159 / 000113464$

17. Barreto CP. Revisão sistemática sobre os efeitos da episiotomia na função sexual da mulher no pós-parto [Dissertação]. São Paulo: Escola de Enfermagem da
Universidade São Paulo; 2014

18. Klein K, Worda C, Leipold H, Gruber C, Husslein P, Wenzl $R$. Does the mode of delivery influence sexual function after childbirth? J Womens Health. 2009; 18(8):1 227-31. doi: 10.1089/iwh.2008.1198

19. Blomquist JL, McDermott K, Handa VL. Pelvic pain and mode of delivery. Am J Obstet Gynecol. 2014;210(5):423. doi: 10.1016/i.ajog.2014.01.032 